\title{
A Fully Coaxial Tunable Broadband Power Equalizer for an X-band MPM
}

\author{
Patrizia Livreri $^{1 *}$, Fabio Lo Gerfo ${ }^{1}$ \\ ${ }^{1}$ Department of Engineering, University of Palermo, Italy \\ *corresponding author, Email: (patrizia.livreri@unipa.it)
}

\begin{abstract}
Microwave Power Modules (MPM) are high power devices largely used for radar applications. An MPM is a hybrid combination of a Solid-State Power Amplifier (SSPA) as a driver and a Traveling wave tube amplifier (TWT) as the final high power stage. Power equalizer is often used to flat the TWT output power gain owing to its big gain fluctuations in the operating frequency range. This paper deals with an optimal design and development of a fully-coaxial easy-tunable power equalizer for an X-band MPM. The structure utilizes a coaxial waveguide as the main transmission line and a coaxial cavity loaded with absorbing material as the resonant unit. A sensitivity analysis of the coaxial cavity length, the thickness of the absorbing disc and inserted depth of the probe on the attenuation amplitude and resonant frequency is carried out. The transmission characteristic curves and the voltage standing wave ratio curves show a good compromise in terms of transferred power and adaptive matching impedance at the input and output ports. The measured results show that the equalization curve meets the requirements well and prove that this structure is practical and effective.
\end{abstract}

Index Terms—Broadband power equalizer; coaxial; MPM; TWT; X-band.

\section{INTRODUCTION}

Wide-band high-power sources operating at microwave and millimeter wave necessitates more efficient devices [1][2][3]. Microwave Power Module (MPM), a hybrid combination of a SolidState Power Amplifier (SSPA) as a driver and a Travelling wave tube amplifier (TWT) as the final high power stage, is widely used in many potential system applications, such as radar and electronic warfare. The gain equalizer is one of the important parts in MPM, which is used to flatten the output gain of traveling wave tube [4][5][6].

Due to the standardization necessary to keep production costs low, the manufacturer of these equalizers generally designs and produces his own network not considering the problems of the circuits connected to it. As a result, equalizers must be mounted separately, requiring additional 
cables and connectors, which results in greater or lesser compensation than desired.

Since the requests of the users of such devices have become rather demanding, as regards what constitutes acceptable operating characteristics or parameters when such devices are used in apparatuses having ever more rigorous and involved design criteria, the redesign of the device for producing the required output characteristic is prevented by the efficiency of this choice.

To get the advantages of small size, lightness, simple structure and ease of processing, the microstrip circuit is the most used for the realization of the equalizer [7][8][9]. In this case, however, the costs of redesigning the device, depending on the requests of the user, limit its use.

To have the advantages of a high factor of merit, low transmission losses, and a tuning capacity, a coaxial equalizer must be realized [10][11].

However, to be economic, such an equalizer should not show the usual high-cost increases usually associated with device miniaturization. Of course, if a smaller, lighter, and lower-cost microwave equalizer were available with a low Voltage Standing Wave Ratio (VSWR) compared to the normal class of microwave equalizers, its contribution to the art would be evident.

In this paper, the design and realization of a fully coaxial tunable broadband equalizer for an Xband MPM, are reported [12].

\section{STRUCTURE AND ANALYSIS}

The microwave amplitude equalizer design consists of several substructures formed by absorbent coaxial resonant cavities. Many factors, such as the length of the cavity, the size of the cavity and the probe, the materials, the position of the adjustable metal micrometric screw, the attenuation screw, the number of resonators, etc., have been taken into consideration in the design [10]. 


\section{a. Description of substructure}

The schematic of the fully coaxial tunable equalizer is reported in Fig. 1. L1 is the cavity length, L2 the insertion depth of the probe, and $D$ the thickness of the absorbing disc.

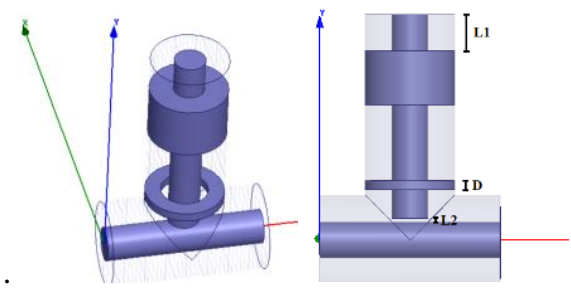

Fig.1. Schematic of the substructure of the designed fullly coaxial equalizer.

One end of the resonator is connected to the main transmission line, whereas the other end is shortcircuited by an adjustable piston. At the base of the resonator, there is a disk of absorbent material whose thickness determines the merit factor Q of the resonant cavity and at the same time has a little effect on the resonance frequency.

The substructure is composed of the following materials:

- Brass: for the resonator, as it has excellent conductivity and a low coefficient of thermal expansion;

- ECCOSORB: for the absorbent disc at the base of the cavity. An absorbent material at microwaves, consisting of polyurethane;

- Copper: for the main transmission line as it has an excellent conductivity and it is very ductile to guarantee the desired diameter;

- Air: for the rest of the structure.

\section{b. Main transmission line size}

The value of the characteristic impedance $\left(Z_{0}\right)$ of a coaxial line section is given by the following equation: 


$$
Z_{0}=\frac{60}{\sqrt{\varepsilon_{r}}} \ln \frac{b}{a}
$$

where: $\varepsilon_{r}$ is the dielectric constant of the filling medium in the coaxial line, whereas $a$ and $b$ are respectively the radius of the internal and external conductor of the coaxial cable.

If $\varepsilon_{r}=1$ (in the case of air), the characteristic impedance value is equal to $50 \Omega$ for a ratio between $\mathrm{b}$ and a approximately equal to $2.3(\mathrm{~b} / \mathrm{a}=2.3)$. For the proposed equalizer, the internal transmission line is made of copper, whereas the external part is air. The values for the radius of the internal conductor and of the external cylinder are $1 \mathrm{~mm}$ and $2.3 \mathrm{~mm}$ respectively as shown in Fig.2.

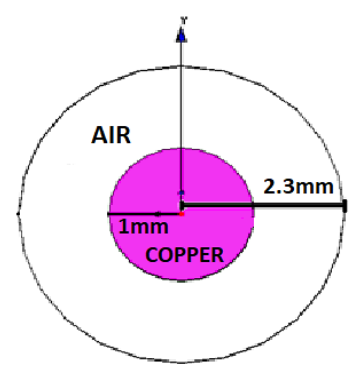

Fig. 2. Internal and external diameters of the main transmission line.

c. Length of the resonator

The length $l$ of the coaxial resonator $l$ is determined from the following relation:

$$
l=(2 n-1) \frac{1}{4} \lambda_{0} \quad \operatorname{con} n=1,2,3, \ldots
$$

where $\lambda_{\mathrm{o}}$ is the resonance wavelength.

Since the resonance wavelength is equal to multiples of $\lambda / 4$, the resonator is called a resonant cavity at $\lambda / 4$.

With reference to equation (6) and considering that the extremes of the operating band are 8 and $12 \mathrm{GHz}$, it is possible to obtain the length of the cavity which in the worst case will be $9.5 \mathrm{~mm}$. 


\section{d. Cavity and probe diameters}

As regards the dimensions of the diameters of the resonant cavity and of the probe, the main factors to take into account are: the maximum power capacity, the dissipation of the conductor, the matching impedance and the propagation of the TEM mode.

For the proposed equalizer, according to the relationship of the characteristic impedance, an external diameter of $4.6 \mathrm{~mm}$ for the cavity and an internal diameter of $2.3 \mathrm{~mm}$ for the probe have been chosen. In relation to this an M5 thread (ISO standard in terms of threads), which corresponds to a diameter of $5 \mathrm{~mm}$ as the external diameter of the cavity and an $\mathrm{M} 2$ thread, which corresponds to a diameter of $2 \mathrm{~mm}$ for the probe, were chosen as reported in Fig.3.

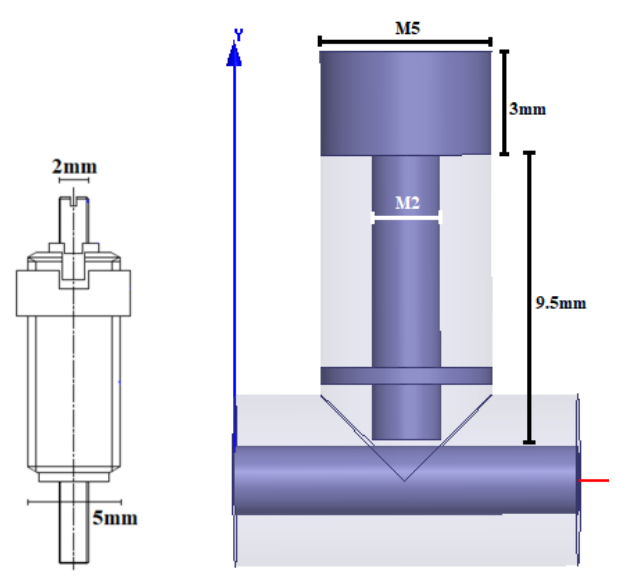

Fig. 3. Size of the tuning screw in the substructure.

e. Absorbing disc thickness

The thickness of the absorbing disc $D$ is directly connected to the thickness of the resonator resistance. So, an increasing of the absorbing disc thickness will increase the resistance $\mathrm{R}$ and according to eq. (1) a decreasing in $\mathrm{Q}$ will occur.

By keeping the length of the cavity and the insertion depth of the probe constant, the transmission coefficient $S_{21}$ and the input VSWR curves vs frequency with the thickness of the absorption cylinder as a parameter have been drawn and shown in Fig.4. It can be observed that the thicker 
the absorption cylinder, the lower the resonance frequency and the attenuation, with a lower input VSWR. A disk thickness of $0.5 \mathrm{~mm}$ as a good compromise between $\mathrm{S}_{21}$ and VSWR has been chosen.



Fig. 4. Transmission and VSWR curves with the thickness of the absorption disk as a parameter.

\section{f. Number of substructures}

The number of secondary structures that must be interconnected to the main line depends on the $\mathrm{Q}$ value of the single substructure, the maximum attenuation range that each of them can provide, the frequency band to be equalized, and the degree of precision with which to adjust the curve. For the proposed equalizer, a 4-substructures was designed. In fact, with the thickness of ECCOSORB chosen $(0.5 \mathrm{~mm})$ and verifying that the maximum value of attenuation that each substructure can give is about $8 \mathrm{~dB}$, the waveform to be given at the input of the amplifier may be equalized in a fairly accurate and precise way. 


\section{SIMULATIONS}

In Fig.5, the schematic design of the proposed equalizer using 4 coaxial cavity resonators in cascade is reported.

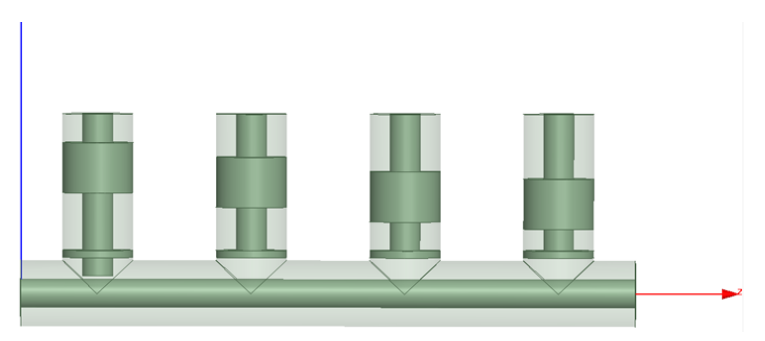

Fig. 5. Schematic design of the designed microwave equalizer structure.

The equalizer has been designed to match the amplifier with the power attenuation curve at each working frequency.

Figure 6 shows the attenuation curves of the designed equalizer, consisting of the sum of the individual attenuation curves of each substructure.

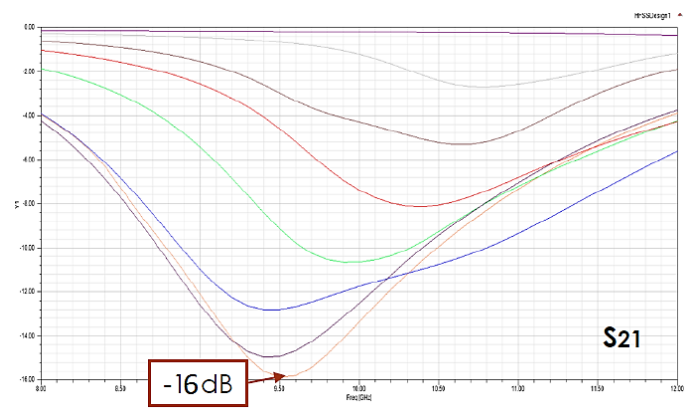

Fig. 6. Equalization curve in the $8-12 \mathrm{GHz}$ band realized by adding the single curves of each resonator.

In Fig. 7, the input and output VSWR of the designed equalizer are shown. The input VSWR has a peak value of about 2.2 , which corresponds to a reflected power of about $13 \%$. 
To keep the VSWR low, an absorbent disc that is not too thin or a probe that is not too coupled have to be use. In both cases this leads to a decrease in attenuation. Since, the desired attenuation is lower than that simulated, then this trade-off can easily be solved.

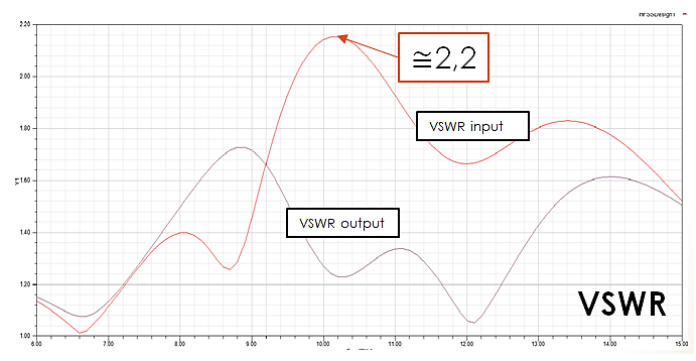

Fig. 7. Input and output VSWR of the designed equalizer.

Starting from the structure shown in Fig.5 and with the help of HFSS by Ansoft, it is possible to model and simulate the single resonator disconnecting all the others.

The simulations of the S21 and VSWR curves have been carried out as follows:

- $L 2$ and $D$ constant and $L 1$ as a parameter, shown in Fig. 8;

$-L 1$ and $D$ constant and $L 2$ as a parameter, shown in Fig. 9.

The curves show a good compromise in terms of transferred power and adaptive matching impedance at the input and output ports.

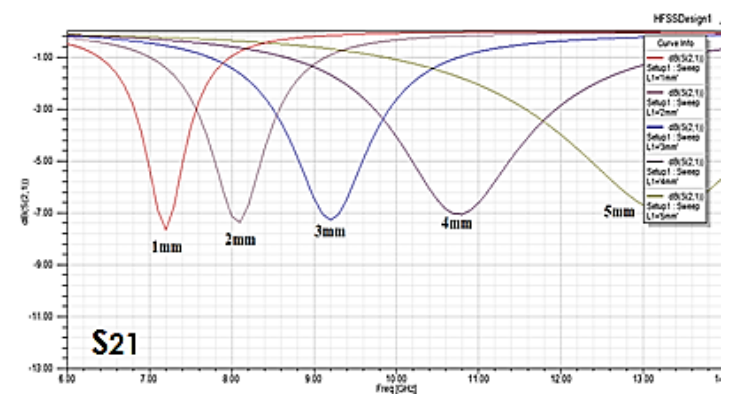




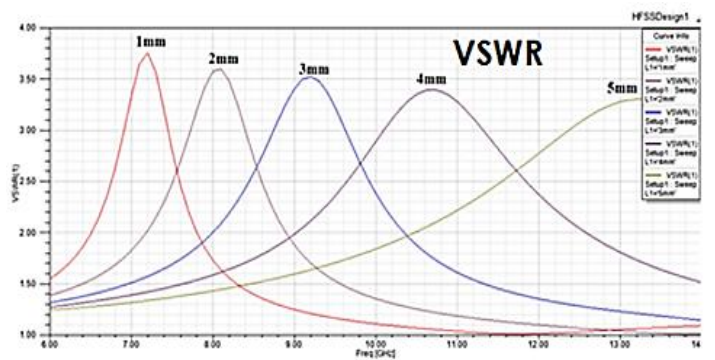

Fig. 8. Transmission and VSWR curves with the cavity length as a parameter.
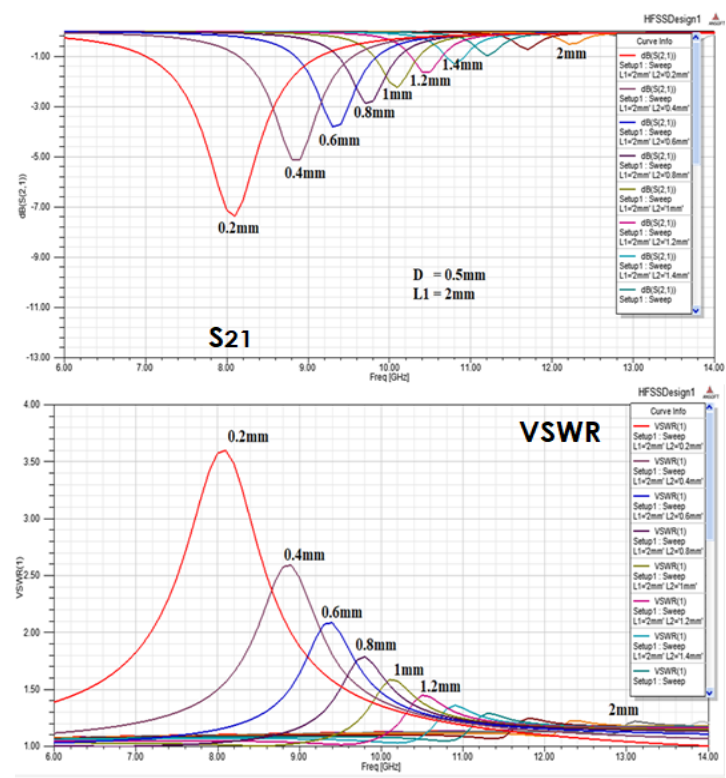

Fig. 9. Transmission and VSWR curves with the probe coupling as a parameter.

\section{MECHANICAL STRUCTURE}

The external design of the device is shown in Fig. 10. It has a height, width, and depth of $15 \mathrm{~mm}$, $9 \mathrm{~mm}$, and $40 \mathrm{~mm}$ respectively. It is made by perforating an aluminum parallelepiped and inserting in it the tuning screws, the absorption disc, and the main transmission line interfaced with the outside through the glass bead.






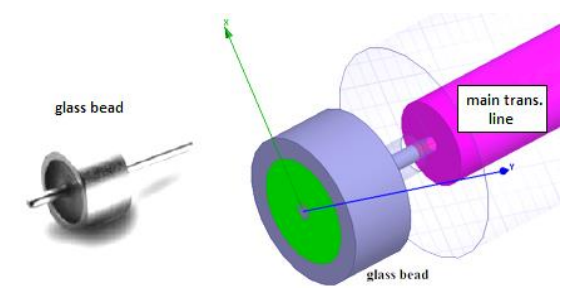

Fig. 10. External design (top) and glass bead for input/output port (down).

The sensitivity analysis of the insertion of the glass bead into the structure and of the distance of the main line between the input and output port, carried out with HFSS, is reported in Fig.11. Figure 11 a) shows the more you insert the glass bead in the structure, the less the attenuation will be. Figure $11 \mathrm{~b}$ ) shows that the more the distance of the main line between the input and output port the more the mismatch will be.



(a)

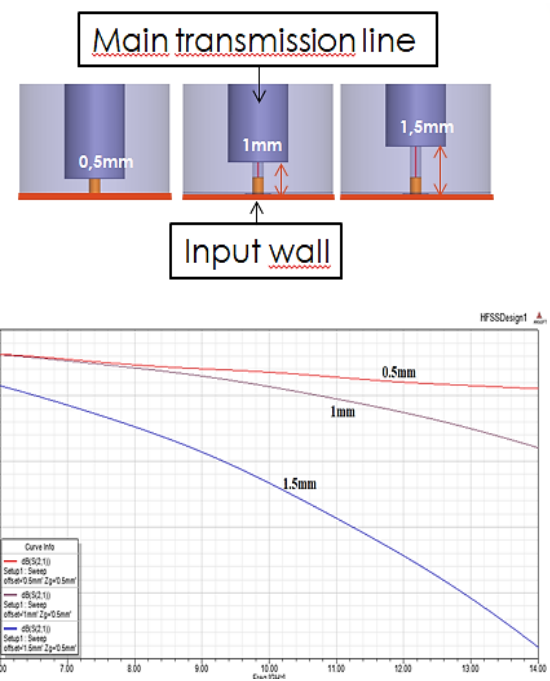

(b)

Fig. 11. Transmission coefficient for different insertion lengths of the glass bead (a) and for different distances of the main transmission line from the input / output port (b). 


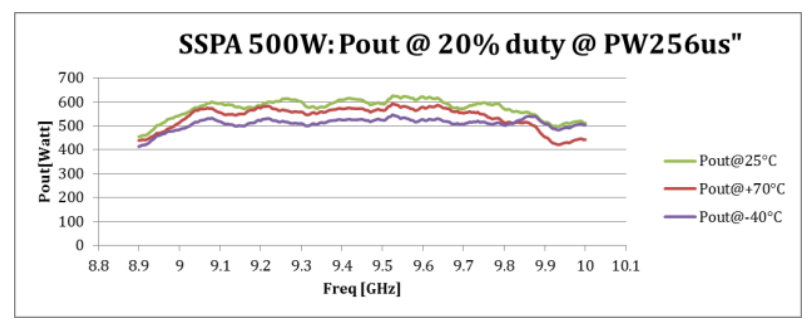

Fig. 15 Output power over Frequency and temperature

\section{CONCLUSIONS}

In this paper, the design of a fully coaxial broadband tunable microwave equalizer was presented. The structure has the advantage of large power capacity, wide operating frequency band, convenient tune and good transmission performance. The equalizer operating at X-band meets the design requirements well to make flat the output characteristic of an MPM for radar applications.

\section{REFERENCES}

[1]. P. Livreri, R. Badalamenti, A. Muratore, "Optimum design and performance of an electron gun for a Ka-band TWT" in IEEE Transactions on Electron Devices, vol. 66, no. 9, pp. 4036-4041, Sept. 2019.

[2]. A. Mistretta, V. Zito, R. Martorana, R. Badalamenti, A. Muratore, P. Livreri, "Design of a 1 kW output power Folded Waveguide TWT operating in ka-band," 2019 IEEE IVEC, Busan, Korea (South).

[3] P. Livreri, V. Zito, A. Muratore, "Design of a double-gap Hughes-type coupled-cavity as a beam-wave interaction structure for a Ka-band Extended Interaction Klystron Amplifier“, 2020 IEEE IVEC, Monterey, CA.

[4] A. V. Konnov, A. V. Malykhin, V. V. Petenkova, G. V. Ruvinskiy, T. I. Chernobay and D. S. Scherbakov, "Analysis with Code Dev. 5.0 of Output Characteristics of Coupled Cavity TWT with Below-Cutoff Sections and with Direct and Inverse Bands Sequence," 2006 IEEE International Vacuum Electronics Conference, Monterey, CA, 2006, pp. 291-292.

[5] T. Ghosh et al., "Mini-TWT in gain and phase matched applications," 2017 IVEC, London, 2017, pp. 1-2, doi: [6] Dong Liang, Wei Han, Weiwei Liu and Xiaoli Jiang, "Research of a compact millimeter-wave gain equalizer," 2016 IEEE International Conference on Microwave and Millimeter Wave Technology (ICMMT), Beijing, 2016, pp. 389-391.

[7]Yu Tang, Ke Wu, Nazih Khaddaj Mallat, Development of Substrate Integrated Waveguide filters for low-cost high-density RF and microwave circuit integration: Pseudo-elliptic dual mode cavity band-pass filters, AEU - International Journal of Electronics and Communications, Volume 70, Issue 10, 2016, Pages 1457-1466, ISSN 1434-8411.

[8]Abu Nasar Ghazali, Mohd Sazid, Srikanta Pal, Dual band notched UWB-BPF based on hybrid microstrip-to-CPW transition, AEU - International Journal of Electronics and Communications, Volume 86, 2018, Pages 55-62, ISSN 1434-8411. 
[9]Jagadish Baburao Jadhav, Pramod Jagan Deore, A compact planar ultra-wideband bandpass filter with multiple resonant and defected ground structure, AEU - International Journal of Electronics and Communications, Volume 81, 2017, Pages 31-36, ISSN $1434-8411$.

[10] Y. Mingshan, Z. Yufeng and N. zhongxia, "Design for Absorptive Coaxial Transmission Line Coaxial Cavity Resonator Microwave Amplitude Equalizer," TENCON 2006 - 2006 IEEE Region 10 Conference, Hong Kong, 2006, pp. 1-3.

[11] G. Jihui, Z. Yinxia, Z. Hong, Z. Xiaomin and Y. Yifei, "The Design of Coaxial Microwave Amplitude Equalizer," 2008 China-Japan Joint Microwave Conference, Shanghai, 2008, pp. 526-529.

[12] M. L. Vecchi, F. Di Maggio, A. Spatola, R. Martorana, A. Mistretta and P. Livreri, "Novel GaN Based Solid State Power Amplifiers, Results, Advances and Comparison with Vacuum Tubes Based Microwave Power Modules," 2018 IEEE 4th International Forum on Research and Technology for Society and Industry (RTSI), Palermo, 2018, pp. 1-6. doi: 10.1109/RTSI.2018.8548356. 